\title{
The Changing Treatment Landscape for Metastatic Urothelial Carcinoma
}

\author{
Presented by Thomas W. Flaig, MD
}

\section{Abstract}

Urothelial carcinoma is the predominant histologic type of bladder cancer. After 30 years of minimal progress in the treatment of advancedstage disease, recent advances in the genomic characterization of urothelial cancer and breakthroughs in bladder cancer therapeutics have rejuvenated the field. Nivolumab, pembrolizumab, atezolizumab, durvalumab, and avelumab are among the exciting recent novel therapeutic advances gaining approvals by the FDA for treatment of advanced-stage urothelial carcinoma. Yet the challenge for clinicians is to determine the optimal choice of agents as first-line or second-line therapy and which offers the best chance for overall survival for the individual patient in this rapidly changing field.

J Natl Compr Canc Netw 2018;16(5.5):636-638 doi: 10.6004/jnccn.2018.0051

"After decades without new bladder cancer treatments, in the past 2 years, and even more so in the past 6 to 9 months, approvals and clinical integration of new drugs have accelerated. We're finally getting traction against the disease," according to Thomas W. Flaig, MD, Associate Dean for Clinical Research, University of Colorado School of Medicine; Chief Clinical Research Officer, UCHealth; and Chair of the NCCN Guidelines Panel for Bladder Cancer. At the NCCN 23rd Annual Conference, Dr. Flaig acknowledged this news and described the systemic treatment options for first- and second-line metastatic urothelial bladder carcinoma (Figure 1). In addition, Dr. Flaig compared the similarities and differences among new immunotherapy options for bladder cancer and reviewed key data supporting their use in patients with metastatic urothelial bladder carcinoma.

Presented by Thomas W. Flaig, MD, University of Colorado Cancer Center, Aurora, Colorado.

Dr. Flaig has disclosed that he has received grant/research support from Agensys, Aragon Pharmaceuticals Inc., Astellas US LLC, AstraZeneca Pharmaceuticals LP, Bristol-Myers Squibb Company, Dendreon Corporation, Eli Lilly and Company, Exelixis Inc., Genentech, Inc., ImmunoTherapeutics, Inc., GTx, Inc., Janssen Pharmaceutica Products, LP, Janssen/Cougar, Medivation, Inc., Novartis Pharmaceuticals Corporation, Pfizer Inc., Roche Laboratories, Inc., Roche/Genentech, sanofi-aventis U.S., Seattle Genetics, SOTIO, LLC, and Tokai Pharmaceuticals, Inc.; has received consulting fees/honoraria from BN ImmunoTherapeutics, Inc. and GTx, Inc.; and has ownership/partnership/principal with Aurora Oncology.

Correspondence: Thomas W. Flaig, MD, University of Colorado School of Medicine, Mail Stop 8117, 12801 East 17th Avenue, Room L18-8117, Aurora, CO 80045. Email: Thomas.flaig@ucdenver.edu
Standard treatment for patients with muscle-invasive, nonmetastatic bladder cancer includes cisplatinbased chemotherapy followed by surgical removal of the bladder or radiation therapy and concomitant chemotherapy. Immunotherapy is emerging as an important treatment for patients with metastatic disease who do not benefit from first-line chemotherapy, and has been added to the updated NCCN Guidelines for Bladder Cancer.

In his presentation on approaches to managing bladder cancer, Dr. Flaig noted that drugs that work in lung cancer also seem to be of benefit in bladder cancer, including platinum-based combinations, gemcitabine, taxanes, and immunotherapy. Dr. Flaig emphasized that "more than 17,000 deaths from bladder cancer are estimated to occur in 2018." In addition, he noted, "75\% of patients are male and many of the patients are or were smokers."

\section{Immune Checkpoint Inhibitors}

Perhaps the most exciting aspect of the recent surge in treatments to manage bladder cancer is the use of immune checkpoint inhibitors (ICIs). "We really needed innovation in bladder cancer," Dr. Flaig said. "Checkpoint inhibitors are a more targeted way to approach late-stage bladder cancer."

Two checkpoint inhibitors (atezolizumab and pembrolizumab) have been used as first-line treatment in pa- 


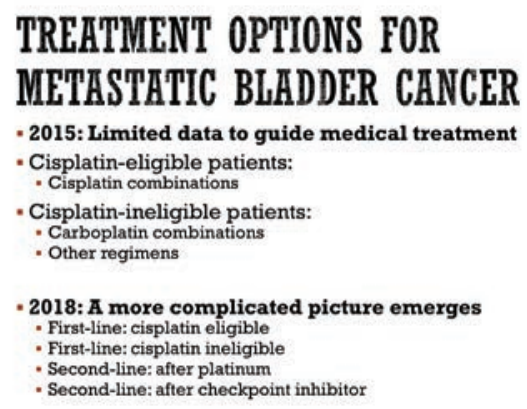

Figure 1. Evolving disease states for the treatment of metastatic bladder cancer.

tients with locally advanced or metastatic urothelial carcinoma who are cisplatin-ineligible. ${ }^{1,2}$ Analysis of clinical trial data can identify 3 broad populations of patients: (1) those who show response initially and continue to show response (responders), (2) those who never show response (innate resistance), and (3) those who initially show response but eventually develop disease progression (acquired resistance). 3,4

Of course, resistance to specific agents does play a role. "ICIs in bladder cancer have been remarkable in a subset of patients, but there are still concerns about safety," Dr. Flaig noted. Although resistance routinely develops in patients treated with conventional cancer therapies and targeted therapies, durable responses suggestive of long-lasting immunologic memory are commonly seen in a subset of patients treated with ICIs. ${ }^{5}$

As for adverse events associated with these checkpoint inhibitors, nephritis, colitis, and pneumonitis have been seen. In addition, thyroid dysfunction and pruritus have been noted with more frequency. "Many patients will have minimal or no significant side effects from these new immunotherapy agents, but a minority will develop significant immune-related adverse events," emphasized Dr. Flaig.

\section{Pembrolizumab}

Based on interim results from the phase III KEYNOTE-045 trial in patients with locally advanced or recurrent urothelial cancer, pembrolizumab sustained its improvement in overall survival (OS) compared with chemotherapy in a 2-year update presented at the 2018 Genitourinary Cancers Symposium. This trial of 542 patients compared pembrolizumab (200 mg every 3 weeks) with chemotherapy (vinflunine, paclitaxel, or docetaxel). ${ }^{6,7}$ The overall response rates were higher with pembrolizumab than with chemotherapy ( $21 \%$ vs $11 \%)$. Most of the responses were in the first 6 months. The most common adverse reactions reported for at least $20 \%$ of pembrolizumab-treated patients included fatigue, musculoskeletal pain, pruritus, decreased appetite, nausea, diarrhea, constipation, and rash. ${ }^{8}$

\section{Atezolizumab}

Dr. Flaig reviewed data from a randomized phase III study of atezolizumab (1,200 mg) every 3 weeks versus chemotherapy (vinflunine, paclitaxel, or docetaxel) in 931 patients with metastatic urothelial carcinoma who experienced disease progression after platinum-based chemotherapy. "Interestingly," commented Dr. Flaig, "atezolizumab did not increase the OS in [patients with] PD-L1-overexpressing (immunohistochemistry 2/3) urothelial carcinoma." However, the researchers concluded that atezolizumab showed a meaningful duration of response in a subset of patients.

\section{Immunotherapy Combinations}

Dr. Flaig delved into combining conventional cancer therapies (such as chemotherapy, radiation, or targeted therapy) and ICI therapies. "This approach may improve the response rate and benefit more patients with cancer because of tumor cell death caused by conventional therapies. The activated immune cells then travel to the damaged tumor tissue, and these immune cells' functions could be further enhanced by ICIs."

Of importance looking forward, Dr. Flaig noted, is the group of patients who are less likely to show response to ICIs. "They should be the next targets for clinical evaluation. However, the issue of patient selection is a priority based on the availability of so many similar compounds," he said.

Overall, cancers with the highest mutational burden, such as bladder cancer, seem to benefit the most from immune checkpoint blockade because of the greater T-cell-mediated antitumor immune response elicited by these cancers. ${ }^{10}$

\section{Other Considerations}

Although the respective clinical trials for all the agents in this class have shown that efficacy cor- 
Flaig

relates with PD-L1 expression, responses have also been seen in patients whose tumors tested negative for PD-L1. Although the presence of PD-L1 can be predictive of response, the lack of PD-L1 expression should not preclude the use of these agents at this point of biomarker development because some patients with negative test results have exhibited responses.

Combination therapies to overcome innate resistance by targeting putative mechanisms of immune evasion within the tumor microenvironment are in various stages of development. ${ }^{11,12}$ According to Dr. Flaig, "they are showing promise as a means of personalizing cancer immunotherapy and potentially enhancing immune memory."

More recent novel immunotherapy drugs (eg, ALT-801 [a tumor-targeted interleukin-2] and ALT803 [an interleukin-15 superagonist complex]) have been tested in bladder cancer, with promising antitumor activity. ${ }^{13}$ "As we start to understand the genetic and nongenetic basis of immunotherapy responders," emphasized Dr. Flaig, "we are seeing the potential predictors for such treatment, and these predictive biomarkers will be critical in the rational clinical advancement of these therapy."

\section{National Clinical Trials Network}

The National Clinical Trials Network is an entity that should become familiar to both clinicians and patients. Through this network, newer advanced agents are being discovered for patients with metastatic bladder cancer. Because most patients with stage IV bladder cancer have disease that has already spread and cannot be removed with surgery, systemic treatment is necessary. Clinical trials are available for most stages of bladder cancer. Patients who are interested in participating in a clinical trial should discuss the risks and benefits of clinical trials with their physician.

\section{References}

1. Balar AV, Galsky MD, Rosenberg JE, et al. Atezolizumab as first-line treatment in cisplatin-ineligible patients with locally advanced and metastatic urothelial carcinoma: a single-arm, multicentre, phase 2 trial. Lancet 2017;389:67-76.

2. Balar AV, Castellano D, O'Donnell PH, et al. First-line pembrolizumab in cisplatin-ineligible patients with locally advanced and unresectable or metastatic urothelial cancer (KEYNOTE-052): a multicentre, single-arm, phase 2 study. Lancet Oncol 2017;18:1483-1492.

3. Restifo NP, Smyth MJ, Snyder A, et al. Acquired resistance to immunotherapy and future challenges. Nat Rev Cancer 2016;16:121-126.

4. Kamat AM, Bellmunt J, Galsky MD, et al. Society for Immunotherapy of Cancer consensus statement on immunotherapy for the treatment of bladder carcinoma. J Immunother Cancer 2017;5:68-70.

5. Pardoll DM. The blockade of immune checkpoints in cancer immunotherapy. Nat Rev Cancer 2012;12:252-264.

6. Bellmunt J, De Wit R, Vaughn DJ, et al. Two-year follow-up from the phase 3 KEYNOTE-045 trial of pembrolizumab (pembro) vs investigator's choice (paclitaxel, docetaxel, or vinflunine) in recurrent, advanced urothelial cancer (UC) [abstract]. Presented at the 2018 Genitourinary Cancers Symposium; February 8-10, 2018; San Francisco, California. Abstract 410.
7. Bellmunt J, de Wit R, Vaughn DJ, et al. Pembrolizumab as second-line therapy for advanced urothelial carcinoma. N Engl J Med 2017;376:10151026.

8. US Food and Drug Administration. Pembrolizumab (Keytruda): advanced or metastatic urothelial carcinoma. Available at: https://www.fda.gov/drugs/in formationondrugs/approveddrugs/ucm559300.htm. Accessed April 2, 2018.

9. Powles T, Durán I, van der Heijden MS, et al. Atezolizumab versus chemotherapy in patients with platinum-treated locally advanced or metastatic urothelial carcinoma (IMvigor211): a multicentre, open-label, phase 3 randomised controlled trial. Lancet 2018;391:748-757.

10. Bellmunt J, Powles T, Vogelzang NJ. A review on the evolution of PD-1/ PD-L1 immunotherapy for bladder cancer: the future is now. Can Treat Rev 2017;54:58-67.

11. Carbognin L, Pilotto S, Milella M, et al. Differential activity of nivolumab, pembrolizumab and MPDL3280A according to the tumor expression of programmed death-ligand-1 (PD-L1): sensitivity analysis of trials in melanoma lung and genitourinary cancers. PLoS One 2015;10:e130142.

12. Hu Z, Ott PA, Wu CJ. Towards personalized, tumour-specific, therapeutic vaccines for cancer. Nat Rev Immunol 2018;18:168-182.

13. O'Donnell JS, Long GV, Scolyer RA, et al. Resistance to PD1/PDL1 checkpoint inhibition. Cancer Treat Rev 2017;52:71-81. 